\title{
DQ white-dwarf stars with low C abundance: possible progenitors
}

\author{
C. G. Scóccola ${ }^{1, \star}$, L. G. Althaus ${ }^{1, \star \star}$, A. M. Serenelli ${ }^{2}$, R. D. Rohrmann ${ }^{3, \star \star}$, and A. H. Córsico ${ }^{1, \star \star}$ \\ 1 Facultad de Ciencias Astronómicas y Geofísicas, Universidad Nacional de La Plata, Paseo del Bosque, s/n, (1900) La Plata, Argentina \\ Instituto de Astrofísica La Plata, IALP, CONICET \\ e-mail: [cscoccola; althaus; acorsico]@fcaglp.unlp.edu.ar \\ 2 Institute for Advanced Study, School of Natural Sciences, Einstein Drive, Princeton, NJ, 08540, USA \\ e-mail: aldos@ias.edu \\ 3 Observatorio Astronómico, Universidad Nacional de Córdoba, Laprida 854, (5000) Córdoba, Argentina \\ e-mail: rohr@oac .uncor .edu
}

Received 5 July 2005 / Accepted 1 December 2005

\section{ABSTRACT}

Aims. The present paper focuses on the evolution of hydrogen-deficient white dwarfs with the aim of exploring the consequences of different initial envelope structures on the carbon abundances expected in helium-rich, carbon-contaminated DQ white dwarfs. In particular, the evolutionary link between the DQs with low detected carbon abundances and the PG 1159, extreme horizontal branch, and helium-rich $\mathrm{R}$ Coronae Borealis $(\mathrm{RCrB})$ stars is explored.

Methods. We present full evolutionary calculations that take a self-consistent treatment of element diffusion into account as well as expectations for the outer layer chemical stratification of progenitor stars upon entering the white dwarf regime.

Results. We find that PG 1159 stars cannot be related to any DQ white dwarfs with low C abundances. Instead, we suggest that the latter could constitute the progeny of the giant, helium-rich $\mathrm{RCrB}$ stars.

Key words. stars: evolution - stars: interiors - stars: white dwarfs - stars: abundances

\section{Introduction}

White dwarfs constitute the final evolutionary stage for the vast majority of stars. Standard stellar-evolution theory predicts that most white-dwarf progenitors are post-asymptotic giant branch (post-AGB) stars of low and intermediate masses that enter the hot white-dwarf stage with hydrogen-rich surface layers (DA spectral type). However, about $20 \%$ of all observed white dwarfs are characterized by hydrogen-deficient atmospheres (commonly referred to as DB spectral type or more appropriately non-DA), and their existence has been a bone of contention amongst researchers for many years. The most commonly accepted mechanism by which hydrogen-deficient white dwarfs are formed is one that involves the occurrence of a final thermal pulse during the post-AGB evolution (the born again scenario; see Fujimoto 1977; Schönberner 1979; and Iben et al. 1983, for earlier references; and Blöcker 2001, for a recent review). In recent years, evolutionary channels other than those involving post-AGB evolution that could lead to the formation of hydrogen-deficient white dwarfs have been suggested.

\footnotetext{
* Fellow of CONICET, Argentina.

$\star \star$ Member of the Carrera del Investigador Científico y Tecnológico, CONICET, Argentina.
}

Amongst them, we find the hydrogen-poor, carbon-normal star evolution directly from the extreme horizontal branch (AGB manqué stars such as sdB stars) into the white dwarf state and the evolution of helium-rich supergiants $\mathrm{R}$ Coronae Borealis (RCrB) stars (Schönberner 1996; and De Marco et al. 2002, for a recent reference). In particular, sdB stars are hot evolved stars with stellar masses around $0.5 M_{\odot}$ that populate the extreme horizontal branch. Their envelopes are so thin that the AGB phase of evolution after core helium exhaustion is avoided. Instead, they evolve directly into low-mass white dwarfs (see Fontaine \& Chayer 2005, for a recent summary of their properties).

Within the born-again scenario, most of the residual hydrogen is completely burnt (see Herwig et al. 1999 and Althaus et al. 2005, for modern computations). The remnant evolves into the central star of a planetary nebulae at a high effective temperature $\left(T_{\text {eff }}\right)$ as a hydrogen-deficient, helium-burning object with carbon-rich outer layers; see Althaus et al. (2005) for a complete simulation of the formation and evolution of hydrogen-deficient white dwarfs through a born-again episode. Observational examples of these hydrogen-deficient post-AGB stars are the very hot PG 1159 and their probable progenitors, the Wolf-Rayet type central stars of planetary nebulae having 
spectral type [WC] (Koesterke \& Hamann 1997; Dreizler \& Heber 1998; Werner 2001). Spectroscopic analyses reveal that most of these stars are characterized by surface layers rich in helium, carbon, and oxygen with a typical composition (in mass fraction) of $(\mathrm{He}, \mathrm{C}, \mathrm{O})=(0.33,0.50,0.17)$ (Werner 2001).

The analysis by Dreizler \& Heber (1998) suggests that PG 1159 stars are the direct predecessors of the majority of helium-rich DO white dwarfs, the hot and immediate progenitors of DB white dwarfs. Theoretical calculations that incorporate the effect of element diffusion and winds also predict the existence of a link between the PG 1159 stars and most of the DO's (Unglaub \& Bues 2000). In this context, evolutionary calculations taking time-dependent element diffusion into account (Dehner \& Kawaler 1995; and Gautschy \& Althaus 2002) have shown that, as a result of the gravitational settling of carbon and oxygen, PG 1159 stars are expected to evolve into white-dwarf stars with a superficially helium-dominated double-layered chemical structure. In fact, two different chemical transition zones would characterize the envelope of the PG 1159 descendants: a still uniform intershell region rich in helium, carbon, and oxygen, which are the relics of the shortlived mixing episode that occurred during the last helium thermal pulse, and an overlying pure helium mantle that thickens as cooling proceeds.

Another group of hydrogen-deficient stars are the extreme helium stars (EHes), which are often considered to be related to the cooler helium-rich RCrB stars because of their similar surface composition and luminosities. As recently discussed by Saio \& Jeffery (2002), the EHes could be formed through a merger of a carbon-oxygen white dwarf with a less massive helium white dwarf.

The development of a double-layered chemical structure in hydrogen-deficient white dwarfs requires initial carbon to be abundant throughout the outer layers of the star. A viable route for this to occur is that a final thermal pulse takes place during the post-AGB evolution. However, the existence of DB white dwarfs characterized by a single-layered profile even from the very beginning of their evolution cannot be discounted, if an evolutionary link between the $\mathrm{RCrB}$ or the AGB manqué stars and hot hydrogen-deficient white dwarfs indeed exists.

From the above concerns, it seems quite reasonable to suppose that DB white dwarfs could harbour envelopes with markedly distinct abundance profiles. This issue motivated us to undertake the present investigation, which is essentially focused on DQ white dwarfs, the supposed cooler descendants of DBs. DQ white dwarfs have $T_{\text {eff }}$ values below $13000 \mathrm{~K}$ (Weidemann \& Koester 1995; see also Bergeron et al. 2001) and are characterized by trace amounts of carbon in their atmospheres. Their abundance by number relative to helium, $\log \left(n_{\mathrm{C}} / n_{\mathrm{He}}\right)$, ranges from -7.3 to -1.5 (MacDonald et al. 1998). The origin of carbon in the atmosphere of these stars is thought to be the result of convective dredge-up of the carbon diffusive tail by the helium convection zone (Pelletier et al. 1986; see also Koester et al. 1982). DQ stars are particularly interesting because the wide range of surface carbon abundances they exhibit could be reflecting the existence of hydrogen-deficient white dwarfs with a variety of evolutionary history and formation processes. In connection with this, MacDonald et al. (1998) have suggested that the diversity of $\mathrm{C}$ to $\mathrm{He}$ ratios is due to a wide range of white-dwarf masses. Indeed, under the assumption that DQs have predecessors with single-layered chemical profiles, they propose that those with very high $\mathrm{C}$ to $\mathrm{He}$ ratios are significantly more massive than the typical white dwarf, say $1.0 M_{\odot}$, and that they have envelope masses running from $10^{-4}$ to $10^{-3} M_{\odot}$. Furthermore, they speculate that DQ with the lowest $\mathrm{C}$ to He ratios can be understood by allowing the WD to be less massive than the canonical $0.6 M_{\odot}$.

We reexamine the carbon abundances in DQs in the framework of detailed evolutionary calculations. The present paper is not intended to explain the whole range of observed $\mathrm{C}$ to He ratios, but instead we focus on those DQs with low carbon abundances. This work is motivated by the recent suggestion by Althaus \& Córsico (2004) that DQs with low detected carbon would not fit into the evolutionary connection PG $1159 \rightarrow \mathrm{DB} \rightarrow \mathrm{DQ}$. In this work, we explore alternative evolutionary channels (with realistic initial outer layer stratification) that could lead to DQs with low carbon abundances. The study of the consequences of structurally different initial envelopes for the carbon surface abundances in DQ constitutes the main purpose of our work. It is worth mentioning that Dufour et al. (2005) have presented new theoretical calculations aimed at explaining the monotonic decrease in carbon pollution in cool DQs with decreasing $T_{\text {eff }}$.

In what follows we provide details about the physical ingredients involved in the calculations, as well as the model sequences studied. In Sect. 3 we describe the main results. We conclude the paper in Sect. 4 with a summary of the results and some final remarks.

\section{Input physics and evolutionary sequences}

The evolutionary code used in this work is the DB white-dwarf evolutionary code recently employed in Gautschy \& Althaus (2002) and Althaus \& Córsico (2004), so we refer the reader to those works for details. The important point here is that this code allows us to simulate the white-dwarf evolution in a self-consistent way including abundance changes resulting from time-dependent element diffusion. Our diffusion treatment, based on a formulation for multicomponent gases by Burgers (1969), accounts for gravitational settling and chemical and thermal diffusion for the nuclear species ${ }^{4} \mathrm{He},{ }^{12} \mathrm{C}$, and ${ }^{16} \mathrm{O}$. Diffusion velocities were evaluated at each evolutionary time step. The abundance changes caused by diffusion were treated separately from those resulting from convective mixing. The microphysics included an updated version of the equation of state of Magni \& Mazzitelli (1979), OPAL radiative opacities for arbitrary metallicity (Iglesias \& Rogers 1996) including carbon- and oxygen-rich compositions, up-to-date neutrino emission rates (Itoh 1997), and conductive opacities (Itoh et al. 1994). Convection is treated in the framework of the mixing length theory as given by the ML2 parametrization (see Tassoul et al. 1990). As for expectations for the carbon enrichment during the DQ stage, the treatment of convection is hardly relevant, since the depth reached by the superficial helium convection zone became almost insensitive to the efficiency of convection 
by the time the domain of the DQs was reached. We assumed complete ionization for calculating diffusion velocities. This prevented us from being confident about the predictions of our models for the carbon enrichment in DQs with $T_{\text {eff }} \lesssim 9000 \mathrm{~K}$.

The initial stellar models needed to start our cooling sequences were obtained by means of the same artificial procedure as described in Gautschy \& Althaus (2002). As one of our main purposes was to understand what could be the predecessors of DQs with very low $\mathrm{C}$ abundance, and because different evolutionary channels lead to distinct chemical profiles, we classified the computed sequences according to their initial chemical structure. Hence, we assumed the following types of initial hydrogen-deficient white dwarf configurations:

- As for the first case, which will be hereinafter referred to as double-layered models, we assumed an initial uniform envelope chemical composition of helium, carbon, and oxygen, which is representative of PG 1159 stars, that is, DB white dwarf progenitors that have emerged from a late helium thermal pulse on the early cooling track (Herwig et al. 1999).

- As for the second case, referred to as single-layered models, we assumed envelopes that are a mixture of helium and trace carbon. There, helium is almost completely separated from carbon even from the onset of the hot phases of the evolution. This chemical stratification is appropriate for hot hydrogen-poor white dwarf progenitors such as the extreme helium stars and the RCrB stars. It could also be appropriate for AGB manqué stars. Note that for these models we assumed $X_{\mathrm{C}}=0.001$. This is suitable for the carbon abundance detected in some $\mathrm{RCrB}$, as recently listed in De Marco et al. (2002) (see their Table 5). For our purposes, we did not consider the presence of trace amounts of oxygen in the outer layers.

For both types of sequences we considered models with stellar masses of $0.50,0.60$ and $0.70 M_{\odot}$ and with helium envelope mass $\left(M_{\mathrm{He}}\right)$ in the range $0.001 \lesssim M_{\mathrm{He}} / M_{*} \lesssim 0.02$. The elected $M_{\mathrm{He}}$ range amply covers what is expected for the white dwarf progenitor from stellar evolution theory. This parameter space allows us to explore the dependence of the carbon enrichment at some length. The chemical composition of the core is from Salaris et al. (1997).

Additionally, we computed the full evolution of a $0.49 M_{\odot}$ model from the extreme horizontal branch (EHB) down to the domain of the DQ white dwarfs with the aim of covering the possible evolutionary connection between DB white dwarfs and the AGB manque stars. The outer chemical structure resembles that of a single-layered configuration.

In Table 1 we list the whole set of model sequences to be discussed in this paper. We use a condensed notation to identify a specific sequence. For instance, 06DL1e3 stands for the double-layered $0.6-M_{\odot}$ sequence with a helium envelope of $0.001 M_{*}$ (DL and SL refer, respectively, to doubleand single-layered models). The 05EHB corresponds to the sequence computed from the extreme horizontal branch. For each sequence, we list the stellar mass in solar units, the mass
Table 1. Model sequences employed in our study.

\begin{tabular}{llll}
\hline \hline Sequence & $M_{*} / M_{\odot}$ & $M_{\mathrm{He}} / M_{*}$ & $X_{12 \mathrm{C}}^{\text {sur }}$ \\
\hline 05DL1e2 & 0.50 & 0.01 & 0.36 \\
05DL2e2 & 0.50 & 0.02 & 0.36 \\
06DL1e2 & 0.60 & 0.01 & 0.36 \\
06DL1e3 & 0.60 & 0.001 & 0.36 \\
07DL1e2 & 0.70 & 0.01 & 0.36 \\
07DL1e3 & 0.70 & 0.001 & 0.36 \\
05SL1e2 & 0.50 & 0.01 & 0.001 \\
05SL2e2 & 0.50 & 0.02 & 0.001 \\
06SL1e2 & 0.60 & 0.01 & 0.001 \\
06SL1e3 & 0.60 & 0.001 & 0.001 \\
07SL1e2 & 0.70 & 0.01 & 0.001 \\
07SL1e3 & 0.70 & 0.001 & 0.001 \\
05EHB & 0.49 & 0.08 & 0.03 \\
\hline
\end{tabular}

fraction of helium content ${ }^{1}$ in the star, and the initial abundance (by mass) of surface carbon. In all of these cases, evolution was computed self-consistently with time-dependent element diffusion from high effective temperatures down to below $T_{\text {eff }} 10000 \mathrm{~K}$.

In what follows, we describe the main results of our calculations. Our main interest is concentrated on expectations for the carbon enrichment in DQ white dwarfs. At such advanced stages of evolution, the artificial way our models are generated is not expected to significantly alter the results to be presented. Hence, a detailed treatment of the evolutionary phases prior to the formation of DB white dwarfs is not of primary importance for our purposes.

\section{Evolutionary results}

\subsection{DL and SL sequences}

Throughout the present work we shall assume that convective dredge-up is the mechanism responsible for the observed carbon abundances in DQ white dwarfs. In this section we shall investigate at some length the role played by two issues that are intimately related to the occurrence of convective dredge-up. These are the outer convection zone (hereinafter referred to as OCZ) and the shape of the outer chemical profiles. Specifically, what determines the amount of carbon that eventually will be dredged to the stellar surface are (i) the location of the bottom of the OCZ, and (ii) the presence of a tail of the ${ }^{12} \mathrm{C}$ distribution at the intershell region. We start our analysis by examining the evolution of the OCZ for white dwarf models with different stellar masses and helium contents, and a distinct chemical structure of their envelopes. We then describe the evolution of the chemical profiles and of the $\mathrm{C}$ to $\mathrm{He}$ ratios, comparing what happens in different sequences.

\footnotetext{
${ }^{1} \mathrm{M}_{\mathrm{He}}$ is the mass fraction of helium content in the whole white dwarf. In the case of SL models, He constitutes the envelope, while in the case of DL models, He is distributed in two zones: the pure helium envelope, which thickens as cooling proceeds, and the zone that lies between the latter and the $\mathrm{C}-\mathrm{O}$ core, which consists of a mixture of $\mathrm{He}, \mathrm{C}$, and $\mathrm{O}$.
} 


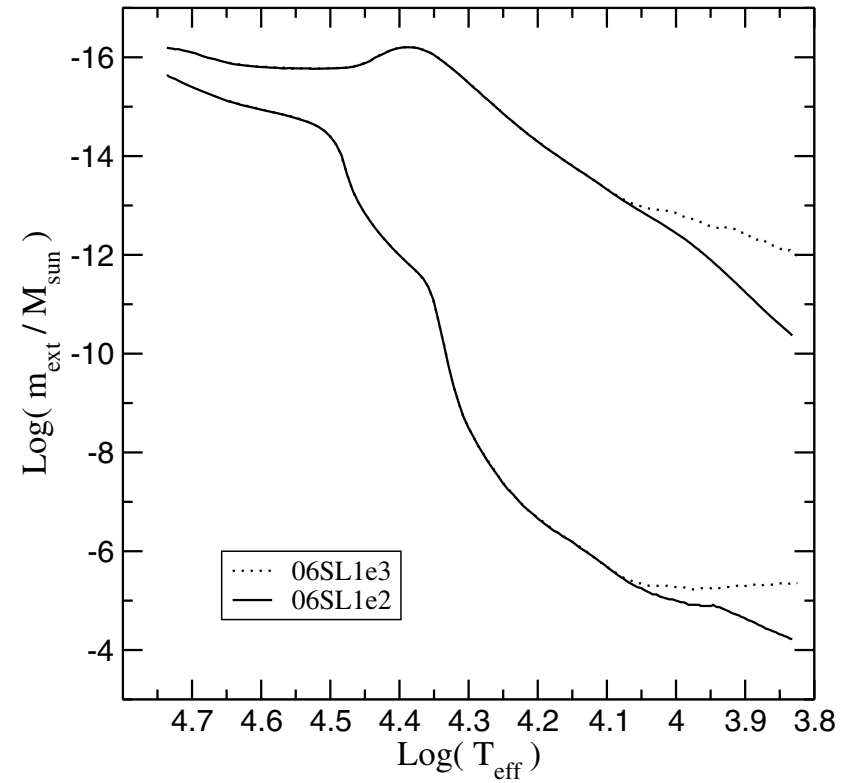

Fig. 1. Top and bottom layers of the OCZ expressed in terms of $\log \left(m_{\text {ext }} / M_{\odot}\right)$ versus $\log \left(T_{\text {eff }}\right)$ (being $\left.m_{\text {ext }}=M_{*}-M_{r}\right)$ for 0.6- $M_{\odot}$ single-layered models with $M_{\mathrm{He}}=10^{-2} M_{*}$ (solid line) and $M_{\mathrm{He}}=$ $10^{-3} M_{*}$ (dotted line). For the model with thinner helium envelope the bottom of the OCZ remains shallower from $T_{\text {eff }} \sim 11200 \mathrm{~K}$.

\subsubsection{Outer convective zones}

In Fig. 1, the boundaries of the OCZ as measured by the outer mass fraction are depicted in terms of the effective temperature for sequences 06SL1e3 and 06SL1e2. These two sequences only differ in the mass of the helium layer. The size of the OCZ is almost identical for both sequences, except when the models reach low effective temperatures, say $\log \left(T_{\text {eff }}\right) \lesssim 4$.1. From there, the bottom of the OCZ for models with thin helium envelopes remains at a fixed maximum depth, whereas the $\mathrm{OCZ}$ of the thick helium envelope continues to grow farther inwards. This is because at low effective temperatures, the convective layers have reached the tail of the carbon distribution and consequently some carbon has begun to be dredged to the surface, causing a rise in the opacity throughout the outer layers. As a result, the temperature gradient increases and the region of partial ionization - which is coincident with the location of the OCZ - rises. This effect, coupled with the fact that the white dwarf is cooling, prevents the OCZ from growing more. On the contrary, the white dwarf models with thick helium envelopes does not suffer from this incipient contamination of carbon, and as the white dwarf cools, the OCZ reaches deeper layers.

Now, we explore the effect of varying the stellar mass, holding the thickness of the helium layer fixed by comparing the OCZ for sequences 06SL1e 3 and 07SL1e3. For effective temperatures below $\approx 22000 \mathrm{~K}$, the base of the OCZ is slightly shallower for the more massive model. This is a consequence of the fact that the stellar envelope is substantially denser for massive models. As a result, conduction becomes the main mechanism to transport energy in those regions, thus inhibiting the occurrence of convection. When we come to compare the evolution of the OCZ for white dwarf models with the same stellar mass and identical helium content, but with a different

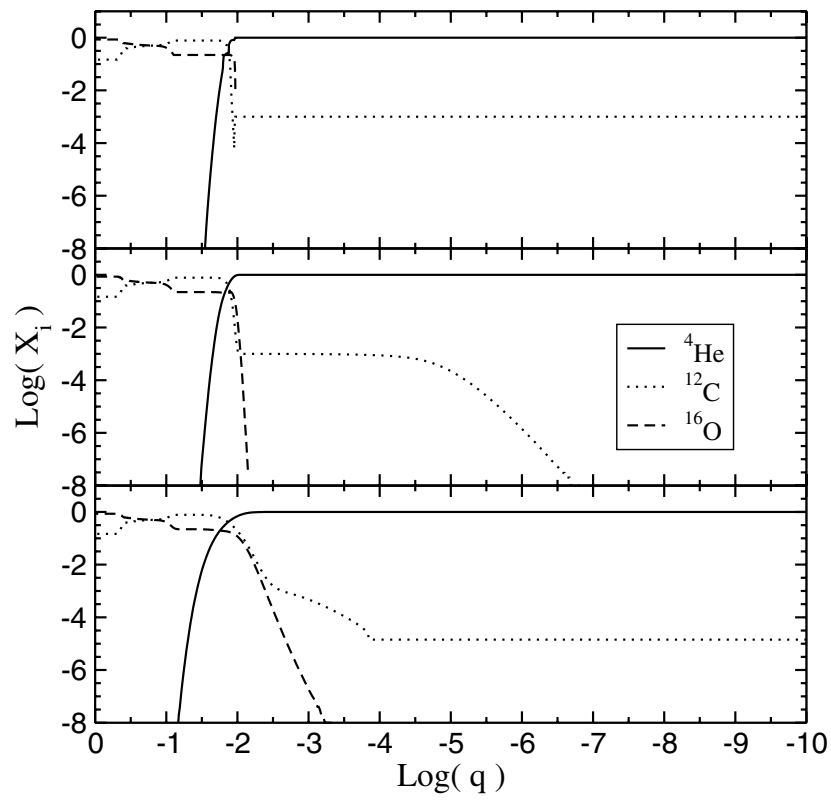

Fig. 2. Abundance (by mass) of ${ }^{4} \mathrm{He},{ }^{12} \mathrm{C}$, and ${ }^{16} \mathrm{O}$ in terms of the outer mass fraction for $0.5-M_{\odot}$ single-layered models with $M_{\mathrm{He}}=10^{-2} M_{*}$. The upper panel shows the situation at the beginning of the sequence. The middle panel depicts the profiles at $T_{\text {eff }}=25700 \mathrm{~K}$, and the bottom panel displays the predictions at $T_{\text {eff }}=7300 \mathrm{~K}$. Note the presence of carbon in the outer layers as a result of convective dredge-up.

chemical structure of their envelopes, the results depend on the mass of the helium layer. In the case of thick helium envelopes, the opacity is larger, since double-layered models have more dredged carbon in their outer layers, and the OCZ is in turn shallower for $T_{\text {eff }} \lesssim 12500 \mathrm{~K}$ as compared to the case of its single-layered counterpart. In the case of the thin envelope, the OCZ is identical for the chemical structure of both single- and double-layered models. This is expected because the thin helium envelope for single-layered models is contaminated with some carbon, and thus the situation is similar to what happens in the case of the double-layered models.

\subsubsection{Evolution of the chemical profiles}

As we shall see, the shape of the initial carbon distribution in the intershell region determines how much carbon will eventually be dredged up to the outer layers. We begin by examining Fig. 2, in which the abundances of ${ }^{4} \mathrm{He},{ }^{12} \mathrm{C}$, and ${ }^{16} \mathrm{O}$ are plotted in terms of the outer mass fraction $q\left[\equiv\left(1-M_{r} / M_{*}\right)\right]$ for $0.5-M_{\odot}$ white-dwarf models with $M_{\mathrm{He}}=10^{-2} M_{*}$ and a single-layered envelope. The figure documents the situation at three different effective temperatures. The top panel corresponds to the initial model of the sequence $05 \mathrm{SL} 1 \mathrm{e} 2$. The envelope is made of a mixture of helium plus carbon traces $\left(X_{\mathrm{C}}=0.001\right.$ by mass). The action of diffusive processes even in deeper layers is already evident in the middle panel, at $T_{\text {eff }}=25700 \mathrm{~K}$. Note that at this stage, carbon has begun to sink into deeper layers due to gravitational settling, whereas at the base of the envelope the helium profile has been smoothed out by chemical diffusion, leading to a non-zero helium abundance in layers originally void of helium. The bottom panel documents the situation at 


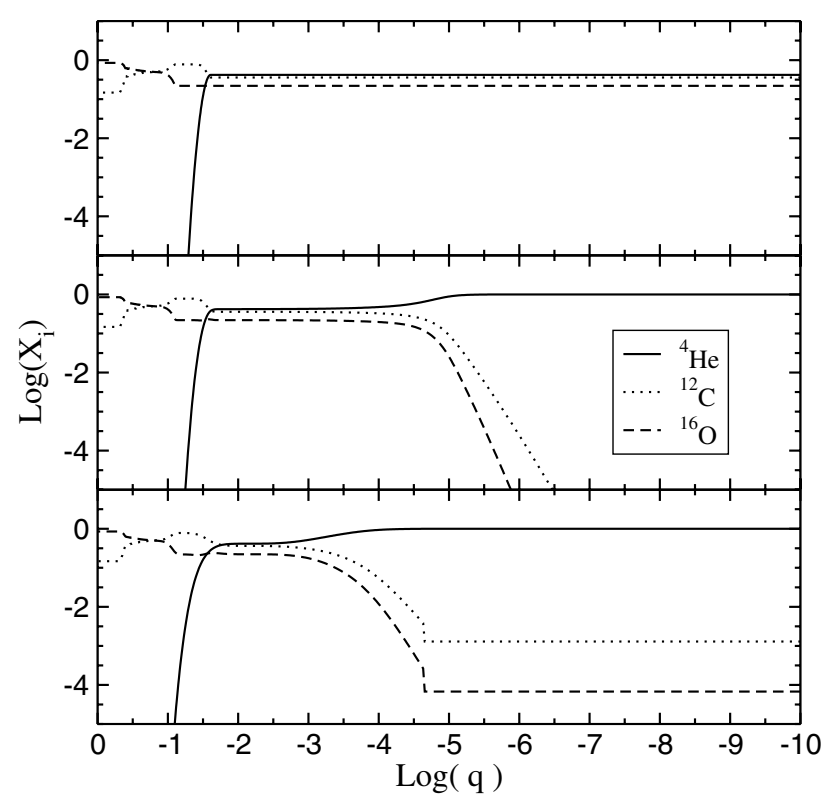

Fig. 3. Same as Fig. 2, but for double-layered models. Upper, middle, and bottom panels depict the situation at 49700, 22800 , and $8900 \mathrm{~K}$, respectively.

$T_{\text {eff }}=7300 \mathrm{~K}$. At low effective temperatures, the OCZ has increased substantially and its base has reached the diffusive tail of the carbon distribution. As a result, considerable amounts of carbon have been dredged up to the surface. Clearly, the final carbon abundance at the surface of the star will be strongly dependent on the shape and extent of the internal carbon profile ${ }^{2}$.

The situation for the corresponding 05DL1e2 doublelayered sequence is shown in Fig. 3. Again, the upper panel corresponds to the initial model of the sequence, before diffusion is allowed to operate. In this case, the envelope consists of a mixture of helium, carbon, and oxygen, with abundances of $0.42,0.36$, and 0.22 by mass, respectively. The overwhelming initial oxygen abundance is due to the occurrence of overshoot episodes during the AGB and post-AGB evolution of the progenitor star (Herwig et al. 1999). The middle panel depicts the situation at $T_{\text {eff }}=22800 \mathrm{~K}$. It is apparent from this plot that carbon and oxygen, abundantly present in the initial outer layer chemical profile, have migrated to deeper layers as an effect of gravitational settling. Clearly, the star has developed a diffusion-induced double-layered structure characterized by a pure helium mantle, which thickens as cooling proceeds, overlying a still uniform intershell region rich in helium, carbon, and oxygen, the relics of the last helium thermal pulse. By the time the domain of the DQ white dwarfs is reached, the thickness of the pure helium mantle amounts to $0.0002 M_{*}$. The bottom panel displays the stage in which, at low effective temperatures $\left(T_{\text {eff }}=8900 \mathrm{~K}\right)$, carbon and oxygen have been dredged to outer layers. At this point, the star should exhibit significant amounts of ${ }^{12} \mathrm{C}$ and ${ }^{16} \mathrm{O}$ in its spectra, in addition to strong features of helium.

${ }^{2}$ We remind the reader that we have assumed carbon to be fully ionized in our treatment of diffusion. This simplification may lead to an overestimation of the surface carbon abundances at such very low $T_{\text {eff }}$ (see MacDonald et al. 1998).

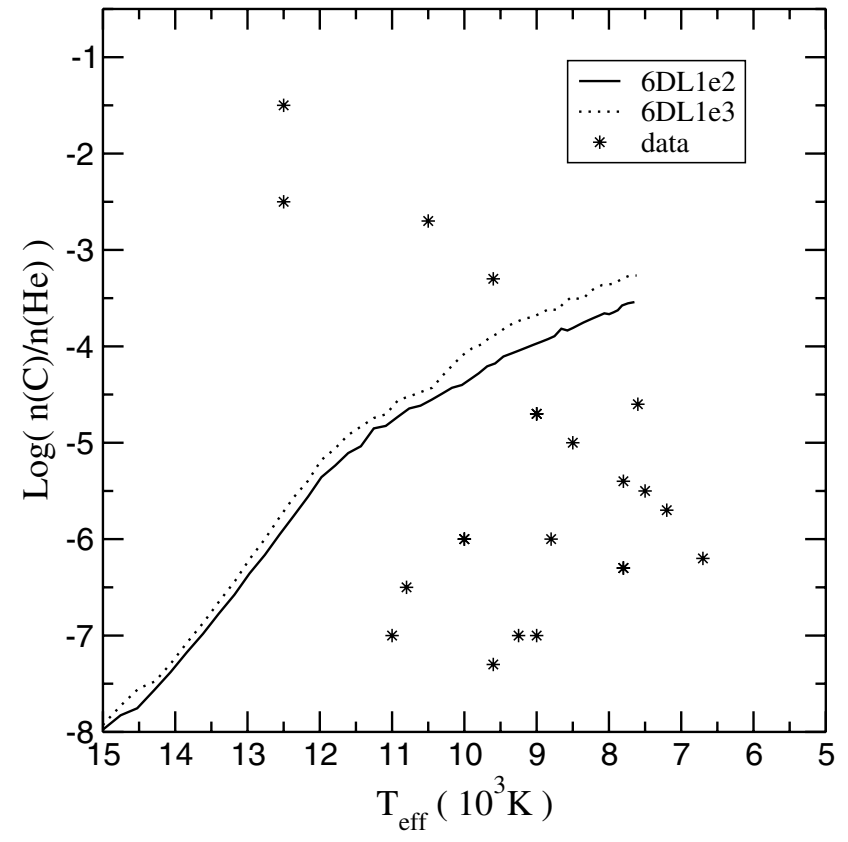

Fig. 4. Evolution of the surface carbon abundance (relative to helium abundance) for 0.6- $M_{\odot}$ double-layered models with $M_{\mathrm{He}}=10^{-3} M_{*}$ (dotted line) and $M_{\mathrm{He}}=10^{-2} M_{*}$ (solid line). Dots represent the observational situation as quoted by Weidemann \& Koester (1995).

\subsubsection{Evolution of surface chemical abundances}

In order to roughly estimate the possible observational consequences of having different initial structures for the outer envelopes (i.e. single- and double-layered envelopes), we now examine the evolution of the surface carbon abundance in terms of $\log \left(n_{\mathrm{C}} / n_{\mathrm{He}}\right)$, with $n_{i}$ the surface number density of the species $i$. We expect the surface carbon abundance to grow as the DQ white dwarfs cool, because more and more carbon should be dredged up to the surface when the OCZ gets deeper. In the single-layered picture, carbon appreciably diffuses from the core to eventually meet the base of the helium convection zone, whilst for the double-layered models, the carbon pollution occurs when the convection zone reaches the tail of the settling carbon. The number density of surface ${ }^{12} \mathrm{C}$ relative to ${ }^{4} \mathrm{He}$ is shown in Fig. 4, for sequences 06DL1e2 and 06DL1e3. Observed photospheric ratios given by Weidemann \& Koester (1995) are also shown in the figure for comparison. For the same stellar mass and initial outer layer chemical stratification (in this case a PG 1159-like profile), we want to see what (if any) the effect of varying the content of helium is. The figure indicates that the carbon abundances are very similar throughout the entire interval of $T_{\text {eff }}$, where $\log \left(n_{\mathrm{C}} / n_{\mathrm{He}}\right)$ is only marginally larger for the thin envelope models. This is due to the fact that for thinner helium envelopes, carbon will reach more external layers than in the case of thicker envelopes. Note that doublelayered models reproduce neither the DQs with low carbon abundances nor those with very high carbon abundances.

The situation becomes quite different if single-layered models are assumed from the start of the evolution. Indeed, note in Fig. 5 that the predicted photospheric abundance of carbon is markedly distinct when single-layered models with 


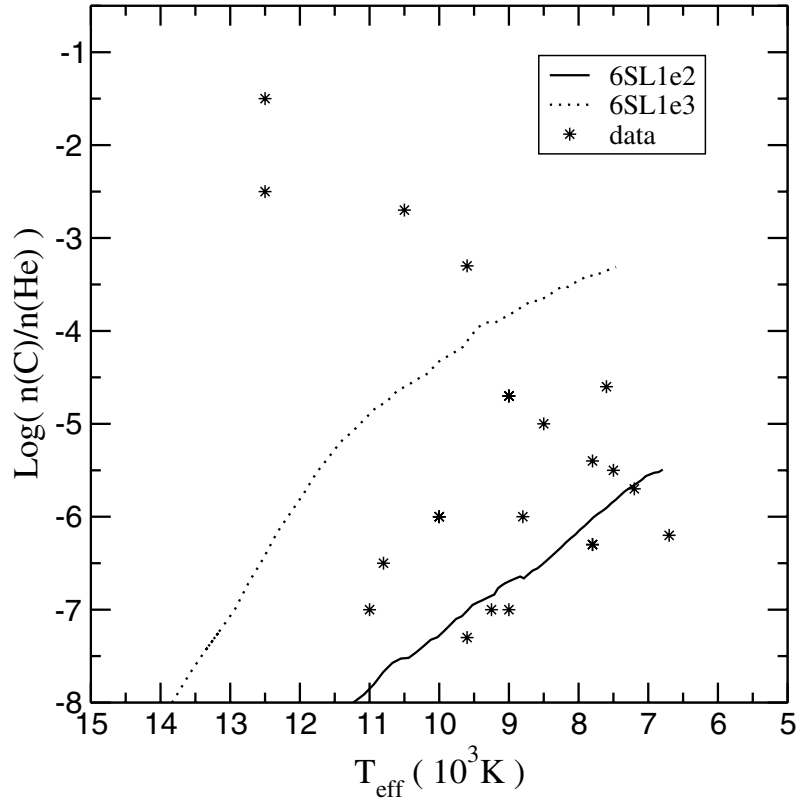

Fig. 5. Same as Fig. 4 but for single-layered models. Remarkably, thin envelope models exhibit a rather large carbon abundance as compared with the case of thick envelope models. See text for additional details.

different helium contents are taken into account. The figure clearly shows that thinner envelope models have a remarkably larger carbon abundances than that of thicker envelope models. Note that for models with $M_{\mathrm{He}}=10^{-3} M_{*}$ the carbon abundance is comparable to what is predicted by their doublelayered counterpart (compare with Fig. 4), whereas for models with $M_{\mathrm{He}}=10^{-2} M_{*}$ the abundance of carbon is exceedingly low and in agreement with the lowest detected carbon abundances. The behaviour displayed in Fig. 5 can be explained on the basis that the envelope initially consists mostly of pure helium in a single-layered model. If the envelope is thick enough, the tail of the carbon distribution is located at such a depth in the star that the amount of carbon that is eventually dredged up by convection is much less than in the case of a thin envelope.

We now turn to the question of whether the stellar mass has any influence on the carbon abundance expected in DQ white dwarfs. In Fig. 6 we show the number density of surface ${ }^{12} \mathrm{C}$ relative to ${ }^{4} \mathrm{He}$ as a function of the effective temperature for three sequences of double-layered models with the same helium content $M_{\mathrm{He}}=10^{-2} M_{*}$ and stellar masses of $0.5,0.6$, and $0.7 M_{\odot}$. We note that the carbon abundance decreases as the stellar mass increases. This behaviour is the one expected, since in more massive white dwarfs conductive transport of energy prevents the OCZ from growing into deeper layers.

We have found that, at low effective temperatures, an appreciable amount of oxygen should emerge at the surface of those DQ white dwarfs whose initial envelope configurations are characterized by a double-layered structure with high oxygen content. This expectation is borne out by Fig. 7, which shows the evolution of the surface oxygen abundance (in terms of the helium one) for a $0.6-M_{\odot}$ model with $M_{\mathrm{He}}=10^{-3} M_{*}$ and a double-layered chemical structure.

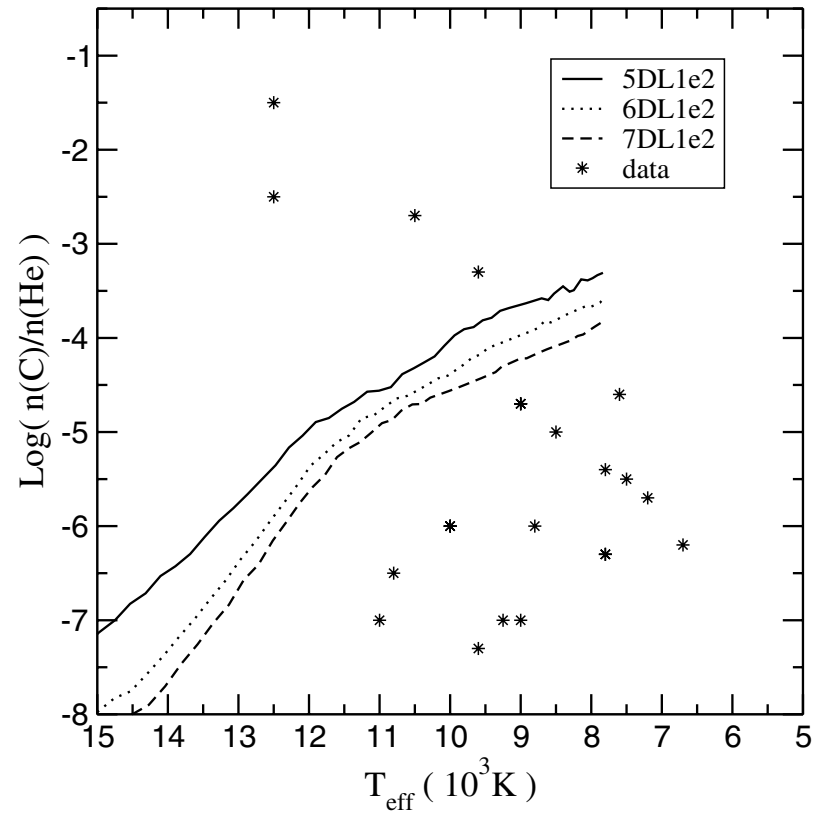

Fig. 6. Same as Fig. 4 but for double-layered models with different stellar masses and equal helium content.

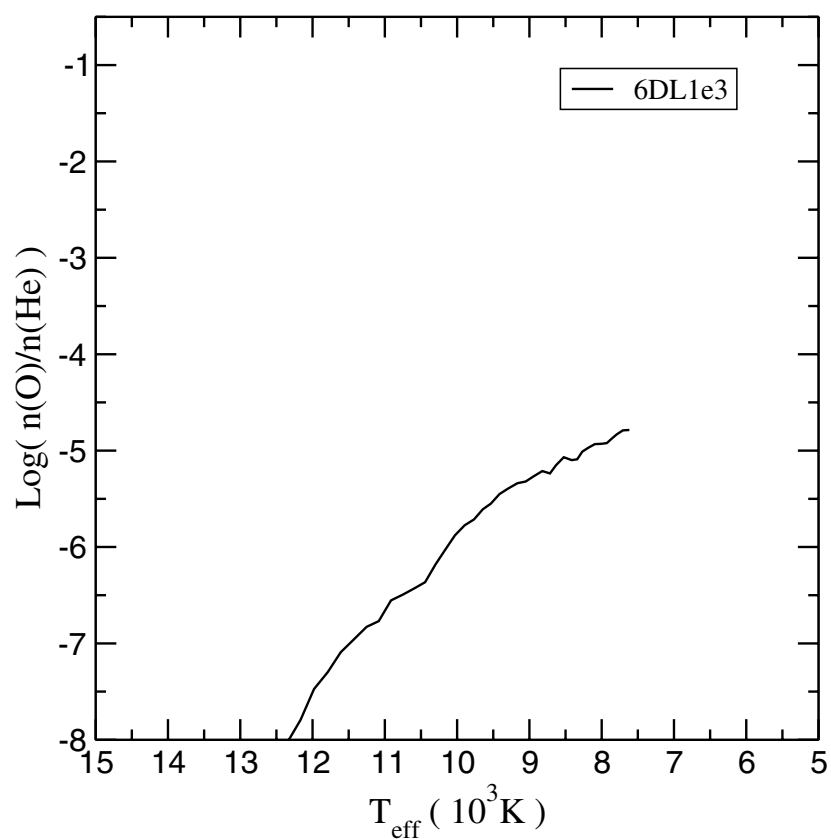

Fig. 7. The evolution of the surface oxygen abundance (relative to helium) for $0.6-M_{\odot}$ models with $M_{\mathrm{He}}=10^{-3} M_{*}$ and a double-layered chemical structure.

\subsubsection{The role of overshooting}

In order to explore the possibility that convective overshoot could alter the expected surface carbon abundances, we considered the occurrence of overshoot mixing below the outer convection zone by employing the simple formalism described in Maeder \& Meynet (1989). In particular, we adopted the distance of overshooting $d_{\mathrm{ov}}$ as a fraction of the pressure scaleheight $H_{\mathrm{p}}$ at the canonical border of the convective zone: $d_{\mathrm{ov}}=$ $\alpha_{\mathrm{ov}} H_{\mathrm{p}}$. We chose the values of $0.1,0.2$, and 0.7 for $\alpha_{\mathrm{ov}}$, and calculated sequences with overshooting for both DL and SL 


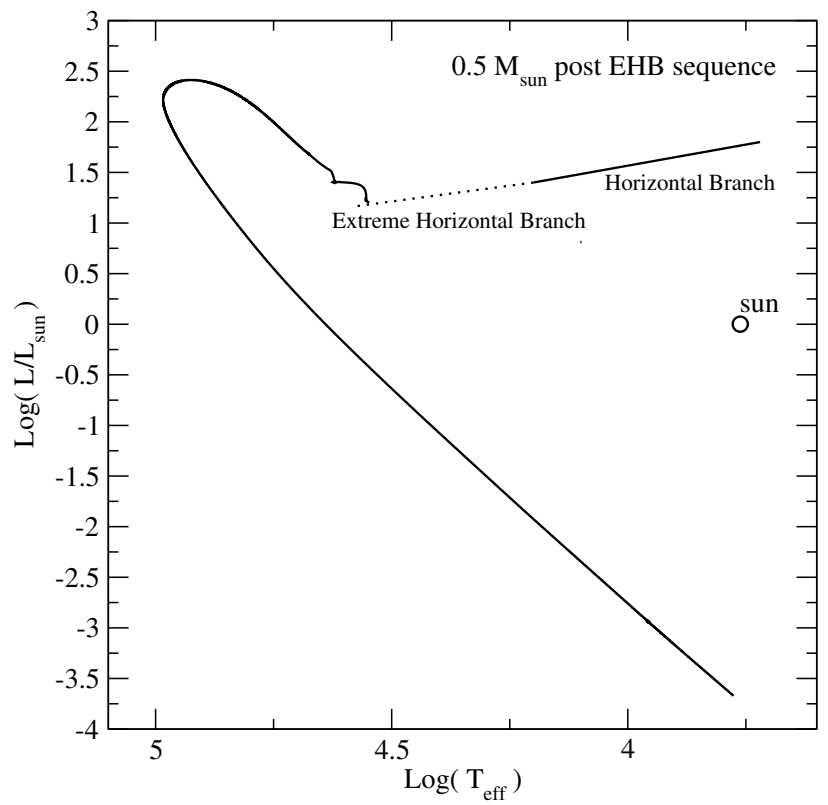

Fig. 8. Evolutionary track for the AGB manqué star. As a reference, the position of the sun is shown with the symbol $\circ$, as well as the location of the horizontal branch. The maximum effective temperature reached is $96500 \mathrm{~K}$ and the highest luminosity is $\log \left(L / L_{\odot}\right)=2.4113$.

and different stellar masses. For all the cases, we find that, as expected, when overshoot episodes are taken into account, the carbon abundance dredged to the surface increases, but only slightly. Indeed, the carbon abundance increases by at most 0.48 dex when a large overshooting is adopted. Needless to say, if overshooting takes place in these stars, a link between DQs with low carbon abundances and PG 1159 stars turns out to be even less probable.

\subsection{The AGB-manqué star}

In what follows, we consider the evolution of a $0.49-M_{\odot}$ star model representative of an AGB manqué star. As mentioned in the introduction, these objects are carbon-normal stars characterized by a very thin hydrogen envelope and evolving directly from the EHB into the white-dwarf state by eluding the AGB stage. For this sequence we consider a hydrogendeficient, helium-rich, carbon-trace $\left(X_{\mathrm{C}}=0.03\right)$ initial stellar configuration. This model was obtained from a $1-M_{\odot}$ main sequence star. We were specially careful in the choice of the initial surface carbon abundance in order to be consistent with observational data as well as with current evolutionary calculations that lead to this type of EHB star. Cassisi et al. (2003) and Lanz et al. (2004) have obtained models of EHB stars by assuming that the core helium flash in low mass stars occurs after the tip of the red giant branch, as a consequence of extreme mass loss episodes. If this flash occurs along the white dwarf cooling regime, the convection zone reaches the hydrogen-rich envelope, mixing hydrogen into the hot heliumburning environment where protons are burned rapidly. As a result, the outermost layers become hydrogen-poor and rich in helium, carbon, and oxygen.

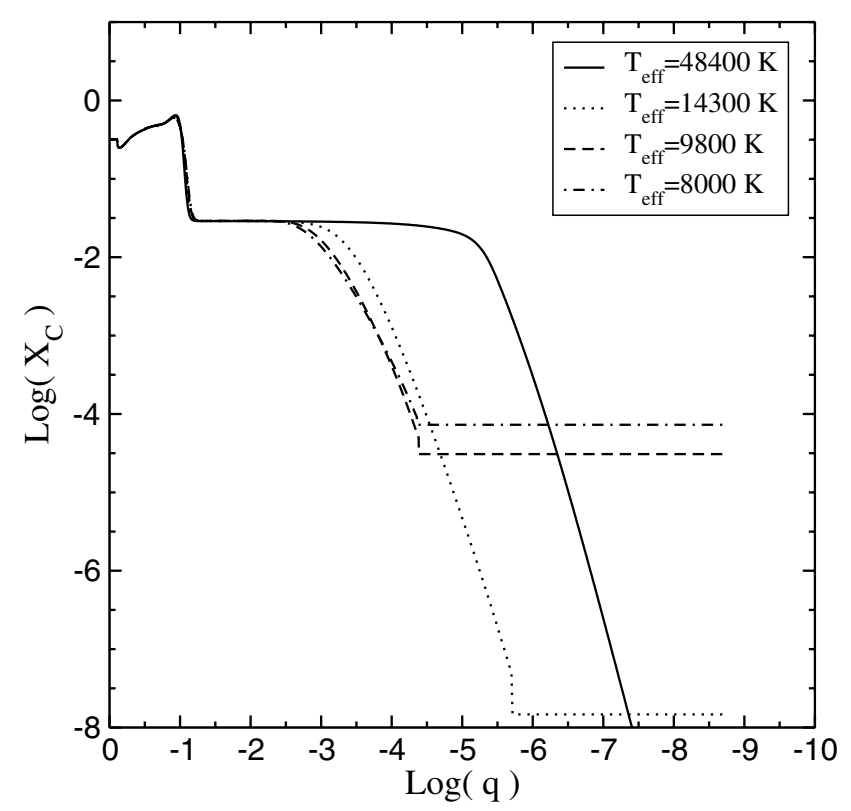

Fig. 9. Abundance (by mass) of ${ }^{12} \mathrm{C}$ in terms of the outer mass fraction for the $0.49-M_{\odot} \mathrm{AGB}-$ manqué model star at four different stages of the white-dwarf cooling.

We followed the evolution of the $0.49-M_{\odot}$ star from the EHB throughout the white-dwarf stage to the DQ regime (sequence 05EHB). In Fig. 8 we show its evolutionary track. Note that the star evolves directly from the horizontal branch to the white-dwarf cooling track. After reaching a maximum effective temperature value, the remmant star enters its final white-dwarf cooling branch. The evolution of the carbon chemical profile is shown in Fig. 9, where we depict the carbon chemical profile at four selected stages. When the DQ regime is reached, the stellar models present a surface carbon abundance that is smaller than in the double-layered models, but larger than that of the singlelayered models, as can be seen in Fig. 10. The latter trend is due to the large initial carbon abundance as compared to the one assumed in single-layered models.

It is important to note that there are observational examples of EHB stars with very low carbon abundances ${ }^{3}$ that cannot be explained by mixing events due to a helium flash or by any current evolutionary scenario. From our preceding results for single-layered models, it is expected that these carbon-poor post-EHB stars could evolve into DQ white dwarfs with very low surface carbon abundances.

\section{Implications of our results}

The DQ stars are evolved helium-rich white dwarfs characterized by the presence of abundant carbon in their outer layers. The origin of carbon in the atmospheres of such white dwarfs has been interpreted as the result of the dredge-up of the diffusive tail of carbon by the superficial helium convection zone. These stars play an important role in the quest for interpreting the spectral evolution in white dwarfs. An interesting feature

\footnotetext{
${ }^{3}$ For example, Lanz et al. (2004) obtained a carbon abundance (by mass) for LB1766 of $10^{-4}$.
} 


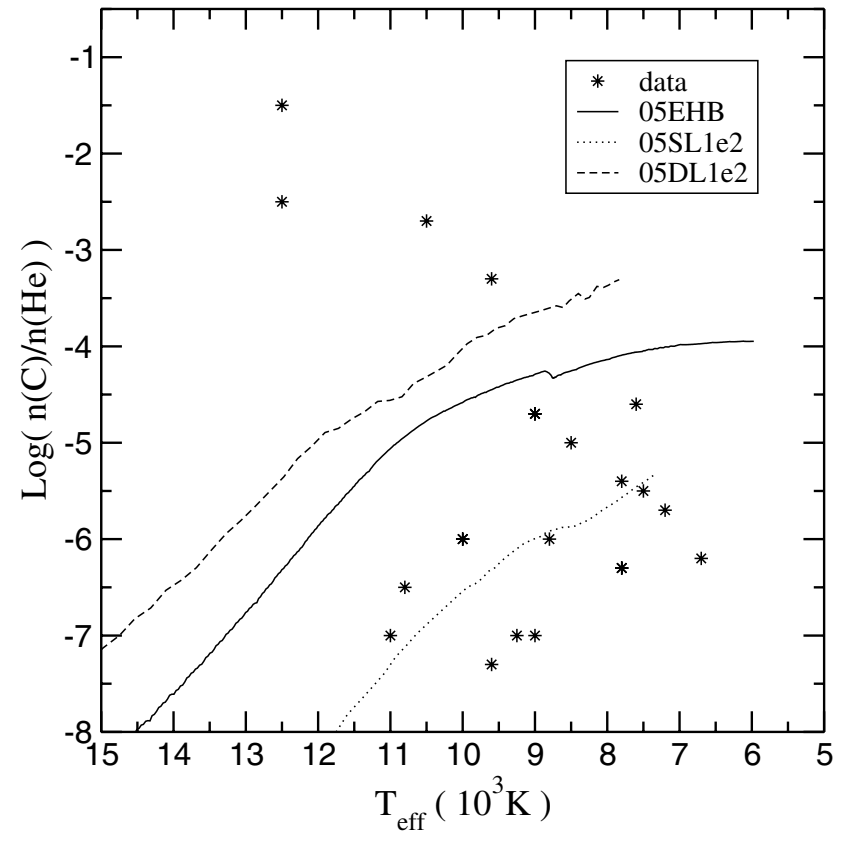

Fig. 10. The surface carbon abundance (relative to helium) for the $0.49-M_{\odot}$ AGB-manqué model. We have also included the carbon abundances for the sequences 05SL1e 2 and 05DL1e2. Also displayed is the observational situation, as quoted by Weidemann \& Koester (1995).

of these stars is that they exhibit a wide range of surface carbon abundances, which could be reflecting a marked diversity in the evolutionary history of the progenitor stars. It is in this context that we undertook this investigation, where our main aim was to explore the implications of the different formation channels for chemical stratifications in the outer layers of DQs. In particular, we tried to establish a connection between the DQs characterized by low carbon abundances and the different evolutionary channels through which hydrogen-deficient white dwarfs could be formed.

Theoretical and observational evidence exists that fosters the plausibility of an evolutionary connection between most of the post-born-again hydrogen-deficient PG 1159 stars with the evolved DQ white dwarfs via the link PG 1159-DB-DQ. Our results strongly suggest that, if the canonical convective dredge-up is the source of observed carbon, those DQ white dwarfs with very low detected carbon cannot be linked to the PG 1159 stars, results which reinforce the conclusions arrived at in Althaus \& Córsico (2004). We have found that this conclusion remains valid irrespective of the stellar mass and helium content with which the PG 1159 stars are formed. This prompts us to suggest that a fraction of the observed DQ population could have followed an alternative evolutionary path that could link them with stars that have somehow avoided the thermally pulsing AGB phase. In addition, we find that PG 1159 also does not constitute a viable route to explain those DQs with very high carbon abundances.

In view of these concerns, we investigated alternative scenarios for the formation of hydrogen-deficient white dwarfs. The hydrogen-poor EHB stars and the supergiant, helium-rich $\mathrm{RCrB}$ stars constitute an attractive possibility. The EHB stars are particularly interesting, because after exhausting helium in their cores, they evolve directly to the white dwarf state. That these stars do not experience the recurrent AGB thermal instabilities is expected to lead to white dwarf progenitors with an outer layer chemical stratification that is quite different from what is expected for a post-born-again PG 1159 star. Indeed, their envelopes resemble one for a single-layered profile. Although the evolution of the AGB manqué star presented here shows a rather high $\mathrm{C}$-to-He ratio, it is important to notice that the initial $\mathrm{C}$ abundance is compatible with evolutionary calculations for the formation of these stars. However, stars do exist in the EHB with very low carbon abundance, which would lead to lower $\mathrm{C}$ to $\mathrm{He}$ ratios. As our results in connection with single-layered models with thick helium envelopes suggest, it is not inconceivable that these stars could be the ancestors of the DQ population characterized by low surface carbon abundance. Also the extreme helium stars (and the associated RCrB stars) evolve directly to the white dwarf regime. These stars have single-layered chemical structure and could evolve into DQ white dwarfs with very low carbon abundances, provided their initial carbon abundance is $\sim 0.001$. An observational counterpart of this expectation is provided by MV Sgr, a hot helium-rich RCrB star characterized by a very low surface carbon abundance (see Table 5 in De Marco et al. 2002).

In closing, we stress again that our conclusions are based on the assumption that the canonical convective dredge-up is the mechanism responsible for the observed carbon abundances in DQ white dwarfs. However, additional mechanisms could be operating at least in hotter helium-rich white dwarfs. As a matter of fact, there is ample evidence that the atmospheres of hot DB white dwarfs are polluted by traces of carbon, which cannot be explained solely in terms of convective dredge-up. For instance, Provencal et al. (2000) present evidence in favour of horizontal motions as a viable mechanism by which inner carbon could be transported to the stellar surface in some hot DBs. Finally, we want to mention that on the basis of chemical homogeneous atmosphere models, we find that a moderate presence of carbon in the outer, radiative layers could hinder the optical detection of high carbon abundances in the convective region. These preliminary results suggest that the relatively low carbon abundances frequently observed in cool He-rich WDs may be explained by a carbon-stratified photosphere, where carbon-rich convective layers remain partially hidden by an enhanced $\mathrm{He}^{-}$opacity in superficial layers $\left(\tau_{\text {Ross }}<1\right)$. We postpone the discussion of this topic to a further investigation.

Acknowledgements. We acknowledge the comments and suggestions of an anonymous referee that greatly improved the original version of this paper. This research was supported by the Instituto de Astrofísica La Plata. A.M.S. was supported by the National Science Foundation through the grant PHY-0070928. A.M.S. is also partially supported by the NSF (grant PHY-0503684), the W.M. Keck Foundation through a grant-in-aid to the Institute for Advanced Study and by the Association of Members of the Institute for Advanced Study.

\section{References}

Althaus, L. G., \& Córsico, A. H. 2004, A\&A, 417, 1115

Althaus, L. G., Serenelli, A. M., Panei, J. A., et al. 2005, A\&A, 435, 631 
Bergeron, P. , Legget, S. K., \& Ruiz, M. T. 2001, ApJS, 133, 413

Blöcker, T. 2001, Ap\&SS, 275, 1

Burgers, J. M. 1969, Flow Equations for Composite Gases (New York: Academic)

Cassisi, S., Schlattl, H., Salaris, M., \& Weiss, A. 2003, ApJ, 582, L43

Dehner, B. T., \& Kawaler, S. D. 1995, ApJ, 445, L141

De Marco, O., Clayton, G. C., Herwig, F., et al. 2002, AJ, 123, 3387

Dreizler, S., \& Heber, U. 1998, A\&A, 334, 618

Dufour, P., Bergeron, P., \& Fontaine, G. 2005, ApJ, accepted [arXiv: astro-ph/0503448]

Fontaine, G., \& Chayer, P. 2005, in Astrophysics in the Far Ultraviolet, ASP Conf. Ser., ed. Sonneborn, Moss, \& Andersson, to be published

Fujimoto, M. Y. 1977, PASJ, 29, 331

Gautschy, A., \& Althaus, L. G. 2002, A\&A, 382, 141

Herwig, F., Blöcker, T., Langer, N., \& Driebe, T. 1999, A\&A, 349, L5

Iben, I. Jr., Kaler, J. B., Truran, J. W., \& Renzini, A. 1983, ApJ, 264, 605

Iglesias, C. A., \& Rogers, F. 1996, ApJ, 464, 943

Itoh, N. 1997, in Advances in Stellar Evolution, Proc. of the Workshop Stellar Ecology (Cambridge Univ. Press), ed. R. T. Rood, \& A. Renzini, 185

Itoh, N., Hayashi, H., \& Kohyama, Y. 1994, ApJ, 436, 418
Koester, D., Weidemann, V., \& Zeidler-K.T., E. M. 1982, A\&A, 116, 147

Koesterke, L., \& Hamann, W. R. 1997, A\&A, 320, 91

MacDonald, J., Hernanz, M., \& José, J. 1998, MNRAS, 296, 523

Maeder, A., \& Meynet, G. 1989, A\&A, 210, 155

Magni, G., \& Mazzitelli, I. 1979, A\&A, 72, 134

Lanz, T., Brown, T., Sweigart, A., Hubeny, I., \& Landsman, W. 2004, ApJ, 602, 342

Pelletier, C., Fontaine, G., Wesemael, F., Michaud, G., \& Wegner, G. 1986, ApJ, 307, 242

Provencal, J. L., Shipman, H. L., Thejll, P., \& Vennes, S. 2000, ApJ, 542,1041

Saio, H., \& Jeffery, C. S. 2002, MNRAS, 333, 121

Salaris, M., Domínguez, I., García-Berro, E., et al. 1997, ApJ, 486, 413

Schönberner, D. 1979, A\&A, 79, 108

Schönberner, D. 1996, in Hydrogen-deficient stars, ed. C. S. Jeffery, \& U. Heber, ASP Conf. Ser., 96, 433

Tassoul, M., Fontaine, G., \& Winget, D. E. 1990, ApJS, 72, 335

Unglaub, K., \& Bues, I. 2000, A\&A, 359, 1042

Weidemann, V., \& Koester, D. 1995, A\&A, 297, 216

Werner, K. 2001, Ap\&SS, 275, 27 